\title{
Supplementary information: Towards the use of dynamic growing seasons in a chemical transport model
}

\author{
A. Sakalli and D. Simpson
}

1. Site-by-site comparisons of observed and estimated start of growing season (SGS) values. For details of methods, see main text.

Table S1: Comparison of average observed SGS of B. pubescens from the PAN database with estimated SGS using T5, LPJ-CRU, TTM and LAT (EMEP standard) methods

\begin{tabular}{|c|c|c|c|c|c|c|c|}
\hline $\begin{array}{l}\text { Lon. } \\
\left({ }^{\circ} \mathrm{E}\right)\end{array}$ & $\begin{array}{l}\text { Lat. } \\
\left({ }^{\circ} \mathrm{N}\right)\end{array}$ & $\begin{array}{l}\text { Alt. } \\
\text { (m asl) }\end{array}$ & $\begin{array}{l}\text { SGS } \\
\text { Obs. }\end{array}$ & $\overline{\mathrm{LAT}}$ & $\begin{array}{l}\text { LPJ } \\
\mathrm{CRU}^{(\mathrm{a})}\end{array}$ & TTM & $\mathrm{T} 5$ \\
\hline 69.05 & 27.10 & 156 & 160 & 129 & 141 & 173 & 163 \\
\hline 68.40 & 27.38 & 301 & 162 & 129 & 139 & 172 & 160 \\
\hline 68.38 & 23.65 & 301 & 163 & 129 & 141 & 175 & 162 \\
\hline 68.02 & 24.15 & 276 & 157 & 128 & 141 & 168 & 158 \\
\hline 67.73 & 29.60 & 321 & 158 & 128 & 141 & 169 & 158 \\
\hline 67.58 & 24.20 & 336 & 152 & 128 & 132 & 165 & 154 \\
\hline 67.35 & 23.82 & 161 & 150 & 127 & 132 & 164 & 153 \\
\hline 67.02 & 27.25 & 216 & 158 & 126 & 134 & 161 & 150 \\
\hline 66.82 & 28.40 & 196 & 143 & 126 & 137 & 162 & 152 \\
\hline 66.35 & 26.72 & 154 & 141 & 125 & 128 & 157 & 144 \\
\hline 66.30 & 25.00 & 118 & 147 & 125 & 127 & 155 & 143 \\
\hline 64.80 & 26.00 & 26 & 140 & 123 & 122 & 148 & 144 \\
\hline 64.52 & 26.45 & 116 & 153 & 123 & 122 & 147 & 147 \\
\hline 64.23 & 19.77 & 226 & 149 & 122 & 127 & 153 & 147 \\
\hline 63.92 & 23.88 & 41 & 141 & 122 & 120 & 142 & 139 \\
\hline 63.55 & 29.02 & 143 & 139 & 121 & 122 & 147 & 142 \\
\hline 63.50 & 10.87 & 61 & 132 & 121 & - & 142 & 133 \\
\hline 63.07 & 29.82 & 136 & 138 & 121 & 120 & 147 & 142 \\
\hline 63.07 & 21.72 & 6 & 139 & 120 & 120 & 145 & 134 \\
\hline 63.00 & 27.72 & 116 & 137 & 121 & 117 & 143 & 141 \\
\hline 62.77 & 30.97 & 149 & 138 & 120 & 119 & 147 & 142 \\
\hline 62.73 & 25.18 & 161 & 137 & 120 & 117 & 145 & 140 \\
\hline 62.63 & 27.05 & 120 & 136 & 120 & 116 & 144 & 141 \\
\hline 62.60 & 29.72 & 81 & 137 & 120 & 120 & 148 & 142 \\
\hline 62.07 & 24.48 & 136 & 136 & 119 & 114 & 139 & 134 \\
\hline 62.02 & 23.03 & 114 & 138 & 119 & 112 & 138 & 133 \\
\hline 61.80 & 29.32 & 82 & 135 & 119 & 114 & 146 & 141 \\
\hline 61.38 & 25.03 & 121 & 136 & 118 & 113 & 136 & 131 \\
\hline 61.02 & 24.45 & 131 & 133 & 118 & 112 & 135 & 131 \\
\hline 60.88 & 14.40 & 321 & 142 & 117 & 115 & 138 & 138 \\
\hline
\end{tabular}

Continued on NextPage... 
TableS1-Continued

\begin{tabular}{|c|c|c|c|c|c|c|c|}
\hline $\begin{array}{l}\text { Lon. } \\
\left({ }^{\circ} \mathrm{E}\right)\end{array}$ & $\begin{array}{l}\text { Lat. } \\
\left({ }^{\circ} \mathrm{N}\right)\end{array}$ & $\begin{array}{l}\text { Alt. } \\
\text { (m asl) }\end{array}$ & $\begin{array}{l}\text { Obs. } \\
\text { Obs. }\end{array}$ & LAT & $\begin{array}{l}\text { LPJ } \\
\mathrm{CRU}^{(\mathrm{a})}\end{array}$ & TTM & $\mathrm{T} 5$ \\
\hline 60.62 & 26.17 & 31 & 133 & 117 & 110 & 133 & 131 \\
\hline 60.43 & 22.75 & 51 & 136 & 117 & 112 & 135 & 131 \\
\hline 60.38 & 22.55 & 11 & 133 & 117 & 112 & 135 & 131 \\
\hline 60.05 & 23.03 & 16 & 130 & 116 & 111 & 135 & 130 \\
\hline 59.67 & 10.78 & 96 & 129 & 116 & 100 & 126 & 124 \\
\hline 57.23 & 9.92 & 21 & 121 & 112 & 108 & 116 & 118 \\
\hline 57.17 & 14.78 & 181 & 131 & 112 & 91 & 126 & 129 \\
\hline 55.97 & 13.33 & 51 & 122 & 110 & 102 & 120 & 124 \\
\hline 55.87 & 12.50 & 41 & 125 & 110 & 93 & 116 & 115 \\
\hline 55.67 & 12.30 & 31 & 125 & 109 & 92 & 114 & 115 \\
\hline 54.95 & -7.72 & 61 & 85 & 109 & 89 & 100 & 109 \\
\hline 53.78 & 21.58 & 128 & 118 & 107 & 90 & 115 & 125 \\
\hline 53.73 & 9.88 & 14 & 105 & 107 & 91 & 105 & 115 \\
\hline 53.67 & 10.27 & 51 & 116 & 107 & 88 & 105 & 115 \\
\hline 53.65 & 10.20 & 47 & 112 & 107 & 88 & 105 & 115 \\
\hline 53.33 & -6.23 & 31 & 100 & 106 & 88 & 100 & 109 \\
\hline 52.85 & 6.18 & 1 & 113 & 105 & - & 99 & 110 \\
\hline 52.75 & 6.90 & 1 & 114 & 105 & 94 & 101 & 113 \\
\hline 52.38 & -6.93 & 81 & 99 & 105 & - & 98 & 109 \\
\hline 52.38 & 4.63 & 1 & 111 & 105 & 100 & 94 & 100 \\
\hline 52.27 & 5.60 & 1 & 125 & 104 & 92 & 98 & 109 \\
\hline 52.25 & 17.10 & 75 & 97 & 105 & 84 & 105 & 117 \\
\hline 52.22 & 4.63 & 1 & 102 & 104 & - & 96 & 109 \\
\hline 52.20 & 5.97 & 1 & 109 & 104 & 92 & 98 & 109 \\
\hline 52.20 & 13.20 & 43 & 103 & 104 & 82 & 101 & 113 \\
\hline 52.10 & 5.12 & 1 & 102 & 104 & 91 & 96 & 109 \\
\hline 52.00 & 5.97 & 1 & 93 & 104 & 92 & 97 & 103 \\
\hline 51.98 & 5.67 & 26 & 108 & 104 & 90 & 97 & 103 \\
\hline 51.97 & 7.63 & 61 & 106 & 104 & 93 & 100 & 114 \\
\hline 51.97 & 6.22 & 1 & 109 & 104 & 96 & 98 & 109 \\
\hline 51.95 & 6.47 & 1 & 110 & 104 & 96 & 98 & 109 \\
\hline 51.80 & 5.40 & 1 & 97 & 104 & 89 & 97 & 103 \\
\hline 51.73 & 5.13 & 1 & 115 & 103 & 89 & 96 & 103 \\
\hline 51.57 & 5.07 & 1 & 86 & 103 & 92 & 96 & 103 \\
\hline 51.48 & 3.95 & 1 & 111 & 103 & 91 & 94 & 101 \\
\hline 51.32 & 3.62 & 1 & 105 & 103 & 91 & 92 & 103 \\
\hline 51.28 & 3.43 & 1 & 117 & 103 & 93 & 91 & 78 \\
\hline 51.08 & -0.88 & 85 & 107 & 103 & 95 & 95 & 101 \\
\hline 50.98 & 3.80 & 16 & 104 & 103 & 92 & 92 & 103 \\
\hline 50.98 & 13.53 & 361 & 117 & 103 & 86 & 106 & 118 \\
\hline 50.00 & 5.73 & 501 & 113 & 101 & 90 & 109 & 112 \\
\hline 49.77 & 7.05 & 481 & 109 & 100 & 85 & 107 & 112 \\
\hline 49.75 & 6.67 & 266 & 103 & 101 & 86 & 104 & 110 \\
\hline 49.02 & -0.03 & 71 & 123 & 100 & 95 & 88 & 100 \\
\hline 48.82 & 9.12 & 331 & 105 & 99 & 79 & 100 & 110 \\
\hline
\end{tabular}

Continued on NextPage... 
TableS1-Continued

\begin{tabular}{llllllll}
\hline $\begin{array}{l}\text { Lon. } \\
\left({ }^{\circ} \mathrm{E}\right)\end{array}$ & $\begin{array}{l}\text { Lat. } \\
\left({ }^{\circ} \mathrm{N}\right)\end{array}$ & $\begin{array}{l}\text { Alt. } \\
(\mathrm{m} \text { asl })\end{array}$ & $\begin{array}{l}\text { Obs. } \\
\text { Obs. }\end{array}$ & $\begin{array}{l}\text { LAT } \\
\text { LPJ } \\
\text { CRU }\end{array}$ & TTM & T5 \\
\hline 48.72 & 9.22 & 381 & 108 & 99 & 79 & 100 & 110 \\
48.45 & 18.93 & 541 & 113 & 99 & - & 108 & 112 \\
48.40 & 11.73 & 461 & 114 & 99 & 85 & 104 & 116 \\
48.33 & 18.37 & 181 & 114 & 98 & 80 & 97 & 107 \\
48.25 & 16.72 & 151 & 101 & 98 & 81 & 95 & 108 \\
48.25 & 16.37 & 203 & 100 & 98 & 80 & 95 & 108 \\
48.18 & 11.17 & 541 & 119 & 98 & 82 & 107 & 116 \\
48.07 & 7.68 & 266 & 106 & 98 & 79 & 91 & 108 \\
47.95 & 8.52 & 681 & 118 & 98 & - & 110 & 118 \\
47.60 & 19.35 & 221 & 106 & 97 & 79 & 94 & 103 \\
47.33 & 21.13 & 91 & 100 & 97 & 75 & 91 & 101 \\
46.03 & 16.57 & 147 & 105 & 95 & 75 & 89 & 104 \\
45.78 & 19.12 & 91 & 98 & 95 & 75 & 85 & 99 \\
44.37 & 20.95 & 122 & 101 & 93 & - & 84 & 103 \\
43.75 & 18.02 & 1001 & 125 & 92 & 92 & 113 & 120 \\
\hline Notes: (a)'-' indicates no values, due to complications with land/sea \\
overlap and/or topography
\end{tabular}




\section{Experiments with fixed day-requirements}

In the final version of the 'T5' equation, a variable number of days, $D_{u, \mathrm{i}}$, is needed with more than $5^{\circ} \mathrm{C}$ before SGS is attained. As seen in Fig. 2 of the main text, $D_{u, \text { i }}$ can vary between zero to almost 40 days (applicable in warmer climates).

This variable $D_{u, \text { i }}$ formulation made possible a very good fit to the observed SGS, in terms of regression statistics and index of agreement. We illustrate here the results of earlier experiments, in which different fixed values, $D_{u, \text { fix }}$, were used to define the number of days $>5{ }^{\circ} \mathrm{C}$. Figure S1 shows that most fixed-value formulations have great problems in reproducing the observed SGS values. The most succesful version is that with a 15-day requirement, and here the results are rather good, indeed much better than the standard EMEP 'LAT' method. However, we found that the 2-parameter T5 method provided even better results. 




(a) $D_{u}=5 \mathrm{~d}$

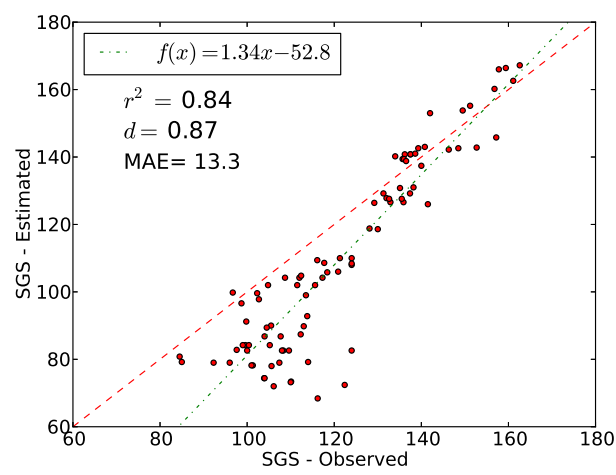

(c) $D_{u}=10 \mathrm{~d}$

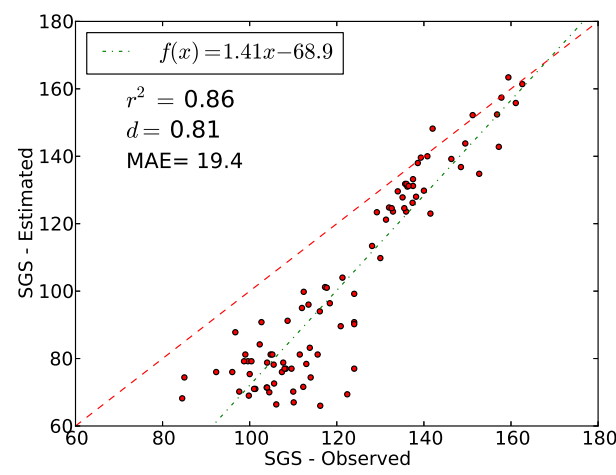

(b) $D_{u}=7 \mathrm{~d}$

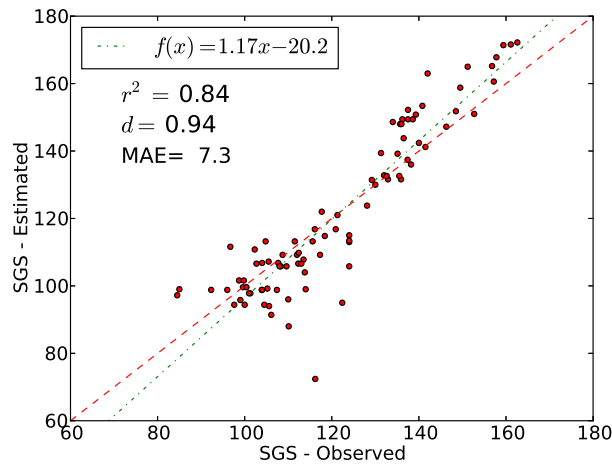

(d) $D_{u}=15 \mathrm{~d}$

Figure S1: Evaluation of preliminary versions of the 'T5' equation, in which fixed $D_{u, \text { fix }}$ values of $5,7,10$ and 15 days were tested. Notation as in main text, Fig. 4. 